\title{
Phytochemical analysis, antimicrobial and antioxidant activities of Aidia borneensis leaf extracts
}

\author{
Zulhamizan Awang-Jamil ${ }^{1}$, Aida Maryam Basri², Norhayati Ahmad ${ }^{1,3}$, Hussein Taha ${ }^{1 *}$ \\ ${ }^{1}$ Environmental and Life Sciences Programme, Faculty of Science, Universiti Brunei Darussalam, Gadong, Brunei \\ ${ }^{2}$ Food Science and Technology Department, School of Applied Sciences and Mathematics, Universiti Teknologi Brunei, Gadong, Brunei \\ ${ }^{3}$ Institute for Biodiversity and Environmental Research, Universiti Brunei Darussalam, Gadong, Brunei
}

\begin{tabular}{l}
\hline ARTICLE INFO \\
\hline Article history: \\
Received on: January 08, 2019 \\
Accepted on: February 07, 2019 \\
Available online: September 10, 2019
\end{tabular}

\section{Key words:}

Aidia borneensis, antibacterial, antifungal, antimicrobial, antioxidant, phytochemicals.

\begin{abstract}
Pharmacological evaluation of Aidia borneensis has not been widely reported yet. This study aimed to evaluate its phytochemical constituents and its antimicrobial and antioxidant activities. Phytochemical compositions of Soxhlet-extracted methanol extract and ultrasonic-extracted aqueous extract were determined by gas chromatography mass spectrometry analysis. Antibacterial and antifungal activities were evaluated by the agar-well diffusion method, and antioxidant activities were determined by 2,2-diphenyl-1-picrylhydrazyl radical scavenging assay. Methanol extract was predominantly characterized by coumaran $(21.12 \%)$ and palmitic acid (6.34\%), and aqueous extract by catechol (15.48\%) and coumaran $(9.13 \%)$. Concentrationdependent antibacterial activities of methanol extract were observed against Bacillus subtilis, Escherichia coli, Pseudomonas aeruginosa, and Staphylococcus aureus, whereas aqueous extract only showed a growthinhibiting effect against $E$. coli. Methanol extract also exhibited antifungal activities against Candida albicans and Saccharomyces cerevisiae but not against Aspergillus brasiliensis. No antifungal activity was shown by the aqueous extract. Aqueous extract revealed significantly higher radical scavenging activity, hence, low $\mathrm{IC}_{50}$ value $(6.0 \mu \mathrm{g} / \mathrm{ml})$, compared to methanol extract $(14.6 \mu \mathrm{g} / \mathrm{ml})$ and the reference standard, Trolox $(9.1 \mu \mathrm{g} / \mathrm{ml})$. This study provides scientific support on the medicinal properties of $A$. borneensis in terms of its phytochemical constituents and its antimicrobial and antioxidant activities.
\end{abstract}

\section{INTRODUCTION}

Aidia borneensis Ridsdale or archer cherry, which is native to Borneo island, is an ethnomedicinal plant under the Rubiaceae family. In Brunei Darussalam, it is locally known as "Sambah bagangan." This plant was initially identified as Aidia racemosa [1] but later, it was re-identifed as A. borneensis [2]. The leaves of this plant are boiled and its decoction is traditionally consumed to relieve body aches or muscle pain and tiredness and is also considered as a remedy for gastric pain [3]. The leaves have been actively harvested by the local villagers for commercial production of herbal tea. To keep up with the local demand, hormonedependent vegetative propagation of this plant has been attempted to effectively mass produce the herbal tea [4]. To the best of

\footnotetext{
*Corresponding Author

Hussein Taha, Environmental and Life Sciences Programme, Faculty of Science, Universiti Brunei Darussalam, Gadong, Brunei.

E-mail: hussein.taha@ubd.edu.bn
}

our knowledge, the scientific evaluation of the pharmacological properties of this plant has not been reported yet, except for its antioxidant property. It was found that the leaf infusion of this plant showed a relatively high antioxidant activity, which could be attributed to its high phenolic, flavonoid, and flavanol contents [5].

This study aimed to provide scientific support for A. borneensis, as it is essential for scientific support to be made available for all ethnomedicinal plants. Moreover, nowadays many ethnomedicinal plants have been considered as significant contributors to modern drug discovery. Therefore, the antimicrobial and antioxidant activities and phytochemical compositions of A. borneensis are reported in this study.

\section{MATERIALS AND METHODS}

\subsection{Leaf Sample Collection}

Leaves of $A$. borneensis were collected from Kiudang forest in Tutong, Brunei Darussalam. The leaves collected were identified 
by Prof. Dato Hj Mohamed Abdul Majid, from the Environmental \& Life Sciences Programme, Faculty of Science, Universiti Brunei Darussalam. After being air-dried for 3 weeks, the leaves were ground into powdered form.

\subsection{Soxhlet Extraction of Methanol Crude Extract}

Following the previous study [6], Soxhlet extraction was used to extract the powdered leaf samples $(50 \mathrm{~g})$ with methanol solvent for 6 hours per day for a total period of 4 days. To remove potential solid residues, the extract was filtered and subsequently evaporated under reduced pressure at $40^{\circ} \mathrm{C}$ to remove the solvent. The solid extract was stored in the dark at room temperature until further use.

\subsection{Ultrasonic Extraction of Aqueous Crude Extract}

Ultrasonic extraction was used, as described elsewhere [6], to obtain the aqueous crude extract. The powdered leaf samples (20 g) were mixed with distilled water in the ratio of $1 \mathrm{~g}$ to $10 \mathrm{ml}$, respectively. Sonica Ultrasonic Cleaner (Soltec, Italy) was used to sonicate the mixture for 30 minutes at $25^{\circ} \mathrm{C}$. It was then filtered to eliminate solid residues, and the filtrate was subsequently lyophilized to produce the solid extract. It was stored in the dark at room temperature until further use.

\subsection{Phytochemical Analysis by GC/MS}

Prior to phytochemical analysis, the crude extracts were dissolved in methanol at $200 \mathrm{mg} / \mathrm{ml}$. Phytochemicals were identified by gas chromatography mass spectrometry (GC/MS), as previously described [6]. Shimadzu QP-2010 GC/MS instrument (Shimadzu Corporation, Japan) was used. Compound identification was done by comparing their retention times and mass spectral data with GC/MS spectral library (National Institute of Standards and Technology library) and only the compounds with at least $90 \%$ similarity with the database were considered.

\subsection{Antimicrobial Assays by Agar-Well Diffusion Method}

Prior to antimicrobial analysis, the crude extracts were re-dissolved in either methanol or distilled water at specific concentrations. As described elsewhere [6], the agar-well diffusion method was used to determine the antibacterial activities of crude extracts. Four bacterial strains, Bacillus subtilis American Type Culture Collection (ATCC)-11774, Escherichia coli ATCC-11775, Pseudomonas aeruginosa ATCC-27853, and Staphylococcus aureus ATCC-29213, and three fungal strains, Aspergillus brasiliensis, Candida albicans, and Saccharomyces cerevisiae were tested.

Mueller-Hinton agar plates were uniformly inoculated with standardized nutrient broth cultures of the bacterial strains and $S$. cerevisiae. However, A. brasiliensis and C. albicans were first cultured in potato dextrose and Sabouraud dextrose broth, respectively, before they were spread uniformly onto their respective agar plates. The agar plates were bored to create wells with 4-mm diameter, in which the crude extract ( $40 \mu \mathrm{l})$ was loaded for testing. For antibacterial positive control, $2 \mu \mathrm{g} / \mu \mathrm{l}$ streptomycin sulfate was loaded, and for negative control, either methanol or distilled water was loaded. The bacterial plates were incubated at $37^{\circ} \mathrm{C}$ for 24 hours, and the fungal plates were incubated at $26^{\circ} \mathrm{C}$ but for 48 hours for $S$. cerevisiae and 72 hours for the other strains. The diameter of the zone of inhibition, where the microorganisms did not grow, was measured.

\subsection{Antioxidant Study by DPPH Radical Scavenging Assay}

The free radical scavenging activities of $A$. borneensis leaf crude extracts were determined by using 2,2-diphenyl-1-picrylhydrazyl (DPPH) assay, according to the methods described previously [7] with some modifications. Briefly, the extract was diluted with its respective solvent (methanol or water) at 100, 50, 25, 10 , and $5 \mu \mathrm{g} / \mathrm{ml}$. The reference standard, 6-hydroxy-2,5,7,Stetramethylchromam-2-carboxylic acid (Trolox), was prepared similarly to serve as the positive control. An aliquot of $800 \mu \mathrm{l}$ of each diluted extract was mixed with $800 \mu \mathrm{l}$ of $0.005 \%$ (w/v) DPPH methanolic solution. For blank, $800 \mu$ of the methanolic DPPH solution was mixed with $800 \mu \mathrm{l}$ of either methanol or water. The mixture was incubated for 30 minutes in the dark and its absorbance (A) was measured at $517 \mathrm{~nm}$. The ability to scavenge DPPH radical was calculated by using the following equation: DPPH radical scavenging activity $(\%)=\left[\left(A_{\text {blank }}-A_{\text {sample }}\right) / A_{\text {blank }}\right] \times 100 \%$

Each experiment was done in triplicate, and the average of three radical scavenging assay (RSA) values at each concentration was calculated. The plot of averaged percentage RSA against extract concentrations was used to produce linear regression line to determine the half maximal inhibitory concentration $\left(\mathrm{IC}_{50}\right)$ value, which is the concentration of the extract that caused a reduction of DPPH initial concentration by $50 \%$.

\subsection{Statistical Analysis}

Results if applicable were presented as mean \pm standard deviation (SD). Significant differences between means were determined using Student's $t$-test. For $p$ value $<0.05$, the difference was regarded as significant, while for $p$ value $<0.01$, the difference was regarded as very significant.

\section{RESULTS}

\subsection{Extraction Yields}

Soxhlet extraction of $A$. borneensis methanol crude extract yielded $8.3 \%$ of extractable components relative to the weight of dried leaf material used. However, for ultrasonic extraction, a higher yield $(20 \%)$ of aqueous extract was obtained. Both crude extracts produced a tea-like aroma.

\subsection{Identification of Phytochemicals}

The GC/MS evaluation of $A$. borneensis methanol and aqueous crude extracts resulted in the identification of eight and five compounds, accounting for $40 \%$ and $31 \%$ of the total relative peak area, respectively (Fig. 1; Table 1).

Based on the peak area, the notable compounds in the methanol extract were coumaran $(21.12 \%)$ and palmitic acid $(6.34 \%)$, whereas, in the aqueous extract, these were catechol $(15.48 \%)$ and coumaran $(9.13 \%)$. Other compounds were also present but at relatively low amounts, with the peak areas ranging from $1.28 \%$ to $3.30 \%$. Comparison of phytochemical constituents showed that 
(A)

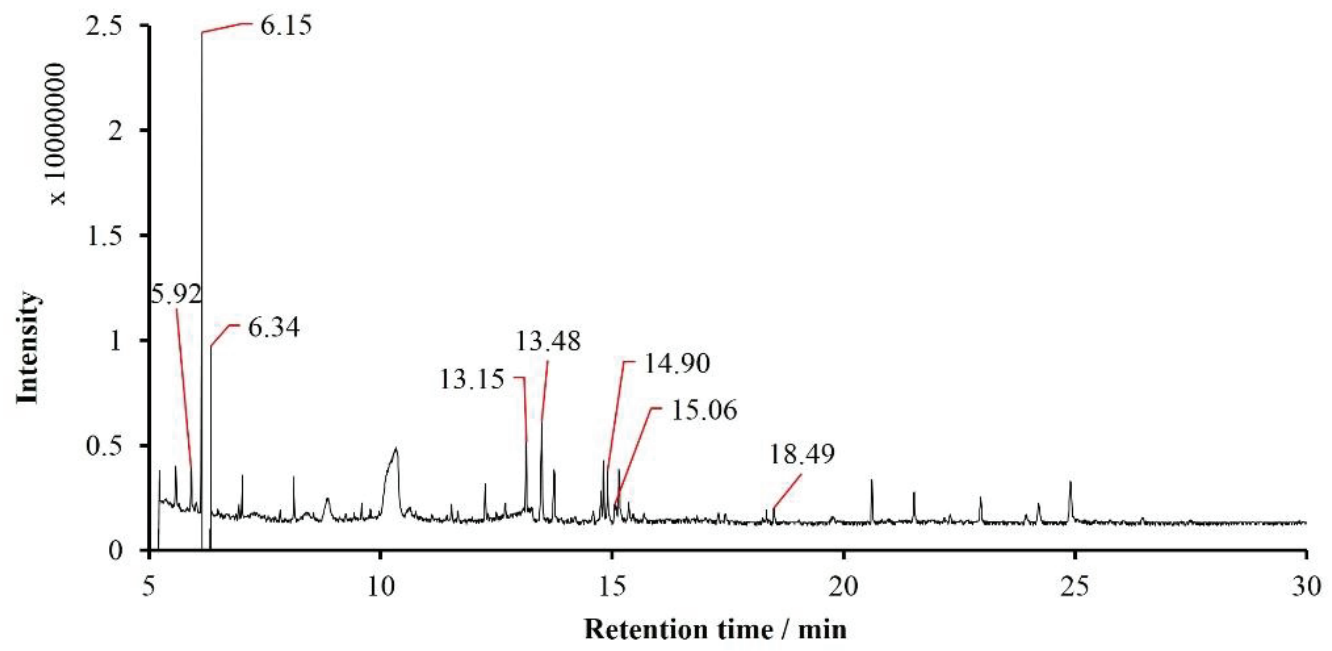

(B)

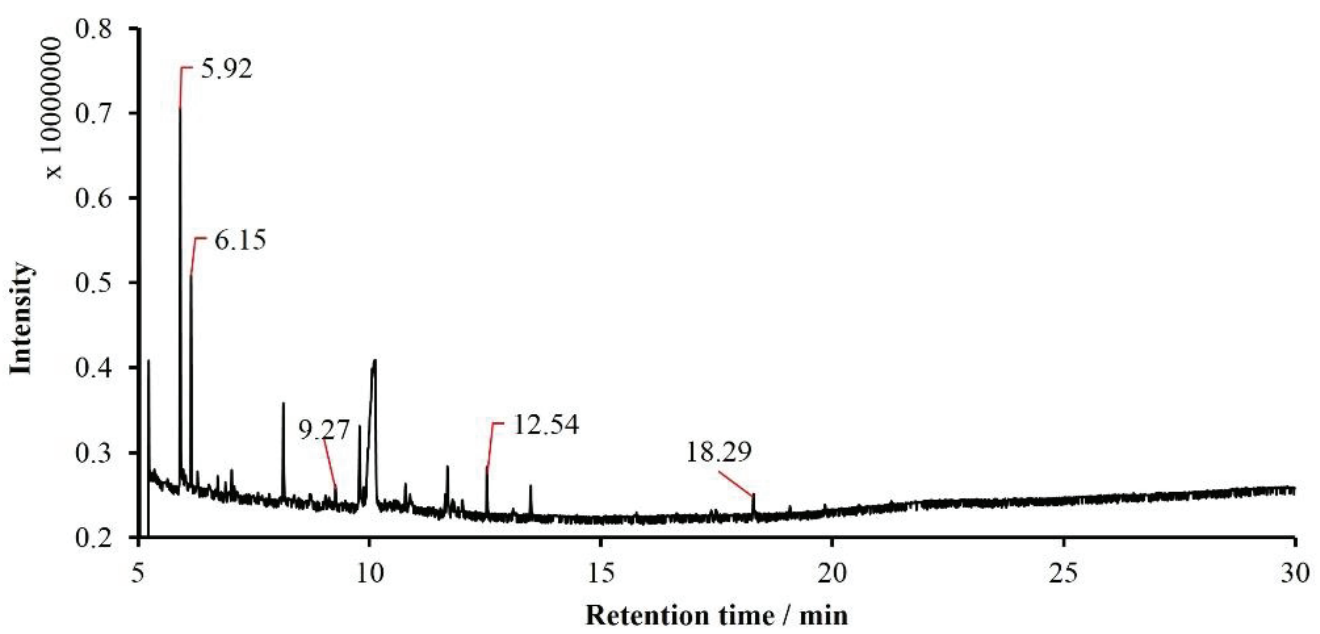

Figure 1. GC/MS spectra of A. borneensis leaf crude extracts. (A) Methanol extract. (B) Aqueous extract. Numbers shown represent the retention times of the peaks analyzed in this study.

Table 1. Phytochemical constituents of $A$. borneensis leaf crude extracts.

\begin{tabular}{|c|c|c|c|c|}
\hline \multirow{2}{*}{$R_{t}$ (minute) } & \multirow{2}{*}{ Compound } & \multirow{2}{*}{ MW } & \multicolumn{2}{|c|}{ Peak area $(\%)$} \\
\hline & & & Methanol extract & Aqueous extract \\
\hline 5.92 & Catechol & 110 & 1.28 & 15.48 \\
\hline 6.15 & Coumaran & 120 & 21.12 & 9.13 \\
\hline 6.34 & 5-Hydroxymethylfurfural & 126 & 2.00 & - \\
\hline 9.27 & 5,6,7,7a-Tetrahydro-4,4,7a-trimethyl-2(4H)-benzofuranone & 180 & - & 1.71 \\
\hline 12.54 & Diisobutyl phthalate & 278 & - & 2.21 \\
\hline 13.15 & Palmitic acid methyl ester & 270 & 3.30 & - \\
\hline 13.48 & Palmitic acid & 256 & 6.34 & - \\
\hline 14.90 & Phytol & 296 & 1.75 & - \\
\hline 15.06 & Stearic acid methyl ester & 298 & 1.68 & - \\
\hline 18.29 & Oxalic acid, 2-ethylhexyl octadecyl ester & 454 & - & 2.11 \\
\hline 18.49 & Mono(2-ethylhexyl) phthalate & 278 & 2.34 & - \\
\hline
\end{tabular}

Compounds are shown in order of retention time $(R)$. Peak area, which represents relative percentage of detected compound in crude extract, and molecular weight (MW) are also shown. Hyphen (-) means the compound was not detected. 
(A)

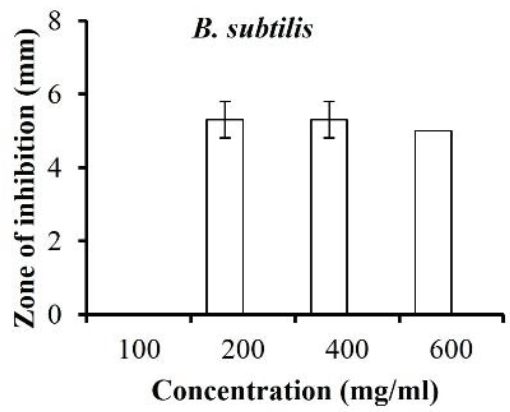

(C)

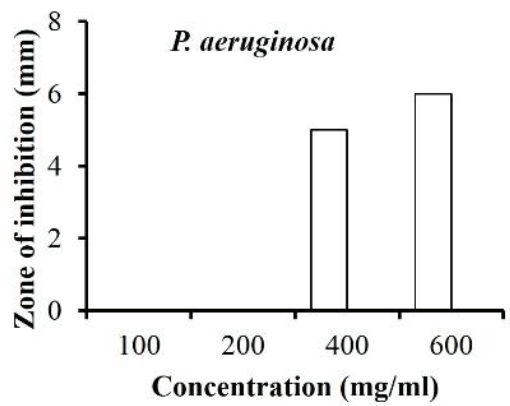

(E)

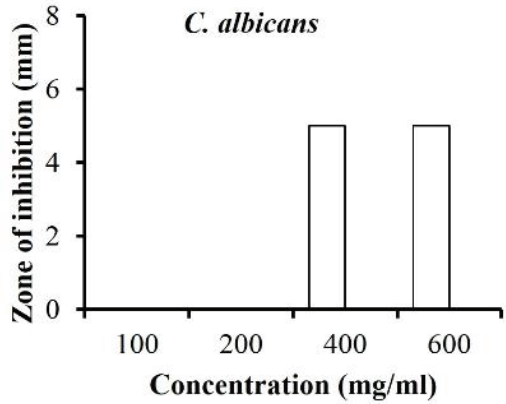

(B)

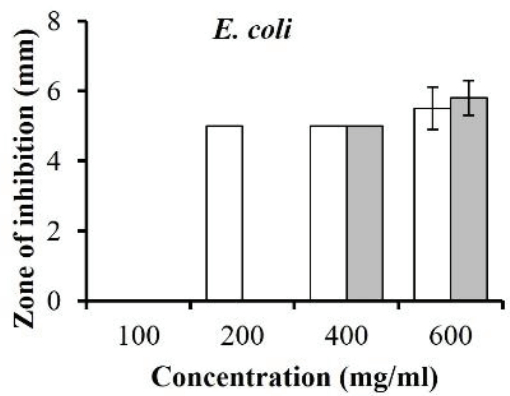

(D)

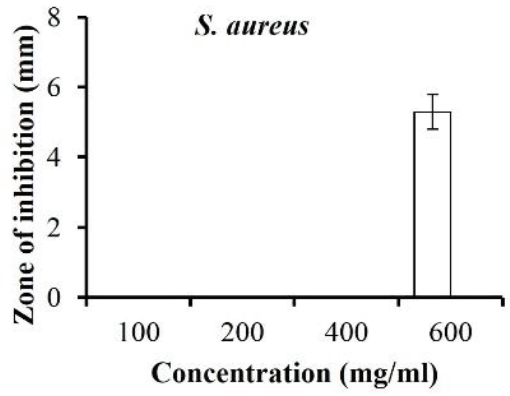

(F)

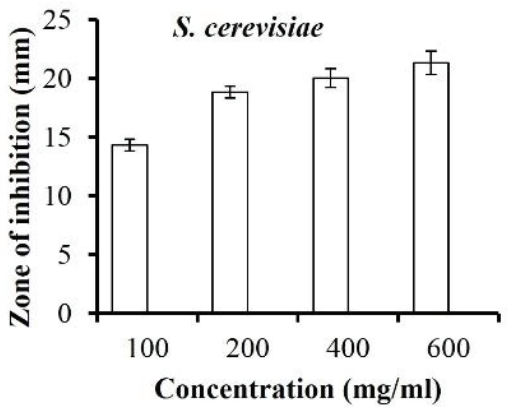

Figure 2. Antimicrobial activities of A. borneensis leaf crude extracts. White bars — methanol extract; Gray bars-aqueous extract. Mean values \pm SD of four replicates are shown. Aspergillus brasiliensis showed no detectable activity for both extracts. Negative control (methanol or distilled water) showed no detectable activity as expected. Positive control $(2 \mu \mathrm{g} / \mu \mathrm{l}$ streptomycin sulfate) had zone of inhibition ranging from 14 to $16 \mathrm{~mm}$.

two compounds (catechol and coumaran) were found in both the methanol and aqueous crude extracts.

\subsection{Antimicrobial Activities of Leaf Crude Extracts}

Antibacterial activities of $A$. borneensis methanol crude extract were observed against Gram-positive (B. subtilis and $S$. aureus) and Gram-negative (E. coli and P. aeruginosa) bacteria (Fig. $2)$. Under the conditions tested, the antibacterial activities were only observed when higher concentrations of the methanol crude extract were used but undetectable at lower concentrations, with $600 \mu \mathrm{g} / \mu \mathrm{l}$ of crude extract showing detectable antibacterial activities against all the bacterial strains tested. At $200 \mu \mathrm{g} / \mu \mathrm{l}$ of the methanol crude extract, the growth of both B. subtilis and $E$. coli was visibly inhibited but not with $P$. aeruginosa and $S$. aureus, suggesting that the crude extract was more potent against B. subtilis and E. coli compared to the other two bacterial strains. The antibacterial activities of $A$. borneensis aqueous crude extract, on the other hand, were less encouraging. No detectable antibacterial activity was observed under the conditions tested, except for $E$. coli, further suggesting that $A$. borneensis was potent against $E$. coli.

Antifungal activities of $A$. borneensis under the conditions tested were only observed with the methanol crude extract but not with the aqueous extract (Fig. 2). This seems to suggest that the methanol crude extract had better potential as an antimicrobial candidate compared to the aqueous extract. The antifungal activities of the crude extract were observed with both yeasts ( $C$. albicans and $S$. cerevisiae) but not with the mould (A. brasiliensis), with $S$. cerevisiae being more susceptible to the methanol crude extract compared to $C$. albicans, as shown with the observed zones of inhibition at 100 and $200 \mu \mathrm{g} / \mu \mathrm{l}$ of the crude extract.

\subsection{Antioxidant Activities of Leaf Crude Extracts}

The DPPH radical scavenging activity was evaluated using a range of different concentrations of the $A$. borneensis extracts and also Trolox for comparison (Fig. 3). The aqueous crude extract generally showed higher radical scavenging activities compared 


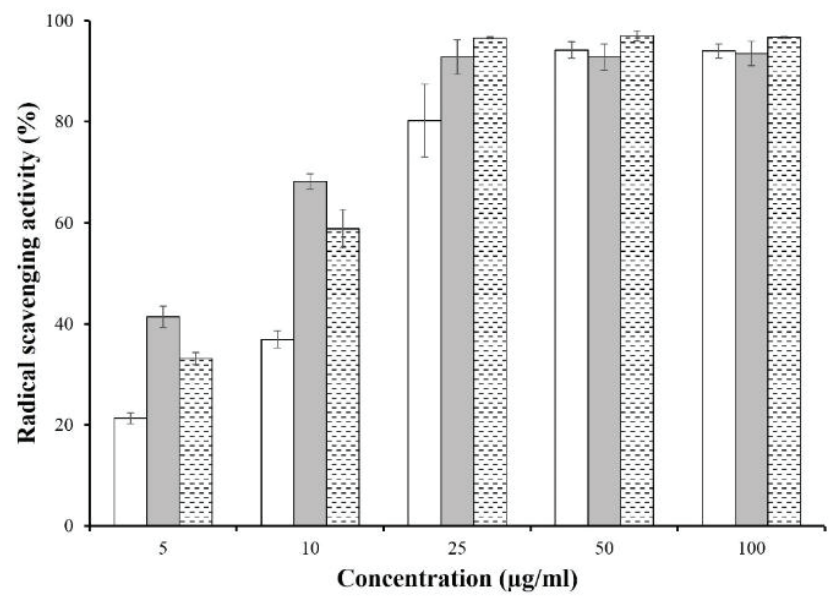

Figure 3. Antioxidant activities of $A$. borneensis leaf crude extracts and Trolox at various concentrations. White bars - methanol extract; Gray bars - aqueous extract; White bars with lines-Trolox. Each value represents the mean $\pm \mathrm{SD}$ of three replicates.

to the methanol crude extract. At both concentrations of 5 and $10 \mu \mathrm{g} / \mathrm{ml}$, the aqueous crude extract exhibited significantly ( $p<$ $0.05)$ higher activities compared to the methanol crude extract, indicating that it was $\sim 2$-fold more active at these concentrations. Moreover, the aqueous crude extract reached its highest activity $(\sim 93 \%)$ at $25 \mu \mathrm{g} / \mathrm{ml}$, whereas the methanol crude extract only showed its highest activity $(\sim 94 \%)$ at $50 \mu \mathrm{g} / \mathrm{ml}$, which again indicated that the aqueous crude extract was more potent in scavenging the hydroxyl radicals. The aqueous crude extract was significantly $(p<0.05)$ more active than Trolox at both concentrations ( 5 and $10 \mu \mathrm{g} / \mathrm{ml}$ ) but had very similar activities ( $p$ $>0.05)$ at the other concentrations $(25,50$, and $100 \mu \mathrm{g} / \mathrm{ml})$.

The $\mathrm{IC}_{50}$ value was determined by plotting percentage RSA against three different concentrations $(5,10$, and $25 \mu \mathrm{g} / \mathrm{ml})$, resulting in the aqueous extract with a linear regression $y=2.36 x$ $+36.06\left(R^{2}=0.9094\right)$, the methanol extract with $y=2.93 x+7.05$ $\left(R^{2}=0.9997\right)$, and Trolox with $y=3.02 x+22.52\left(R^{2}=0.9709\right)$. This resulted in the $\mathrm{IC}_{50}$ values of 6.0 and $14.6 \mu \mathrm{g} / \mathrm{ml}$ for aqueous and methanol extracts, respectively. Higher antioxidant activity is indicated by having a lower $\mathrm{IC}_{50}$ value. Based on these $\mathrm{IC}_{50}$ values, the aqueous crude extract showed significantly $(p<0.01)$ higher antioxidant activity of up to $\sim 2$-fold more active compared to the methanol crude extract. Interestingly, the aqueous crude extract significantly $(p<0.01)$ surpassed the antioxidant activity of Trolox that had an $\mathrm{IC}_{50}$ value of $9.1 \mu \mathrm{g} / \mathrm{ml}$, which highly suggests that the aqueous crude extract has great potential as an antioxidant via radical scavenging activity.

\section{DISCUSSION}

Aidia borneensis crude extracts were both predominantly characterized by coumaran, with $21.12 \%$ and $9.13 \%$ in the methanol and aqueous extracts, respectively. Coumaran is known as a minor component in many plant leaves but has been observed as a major component in Barago officinalis seeds [8]. Coumaran is reported as a potent biofumigant due to its ability in inhibiting acetylcholinesterase [9]. It is also shown to have anti-inflammatory effects [10] and is a promising agent for central nervous system trauma and ischemia due to its potent antioxidant activity [11].

Catechol was also substantially present in the aqueous crude extract $(15.48 \%)$ but not in the methanol extract $(1.28 \%)$. Catechol isolated from Diospyros kaki roots and its structural analogs were shown to have significantly potent antibacterial activities against food-borne bacteria [12]. This suggests that catechol could be the active antibacterial agent against $E$. coli, as observed in this study. However, the unobserved concentrationdependent antibacterial activity against the other three bacterial strains ( $B$. subtilis, $P$. aeruginosa, and $S$. aureus) indicates that the growth-inhibiting effect of $A$. borneensis was likely selective. Previous study showed that catechol at a dose of 5.0 $\mathrm{mg} /$ disc significantly and moderately inhibited the growth of Clostridium difficile and E. coli, respectively, but did not inhibit bifidobacteria and lactobacilli [13].

Catechol was also found to have antifungal activities against Fusarium oxysporum and Penicillium italicum [14]. However, A. borneensis aqueous extract was not found to have any antifungal effect under the conditions tested, showing that $A$. brasiliensis, $C$. albicans, and $S$. cerevisiae were resistant to the aqueous extract. This is due to selective growth-inhibiting effect.

In comparison with the aqueous extract, the methanol extract showed superior antibacterial and antifungal effects. This is perhaps unsurprising because other plants such as Alpinia officinarum also displayed the best biological activities when methanol was used as extraction solvent compared with water [15]. A potential candidate to explain these superior antimicrobial effects in A. borneensis is palmitic acid although other compounds should not be ruled out. This fatty acid was notably present $(6.34 \%)$ in the methanol crude extract but was undetected in the aqueous extract. Palmitic acid methyl ester was also detected although in a lower amount $(3.30 \%)$. Palmitic acid and other fatty acids have been found to exhibit antimicrobial activities against various oral microorganisms, including C. albicans [16]. Antifungal activities of palmitic acid against selected phytopathogenic fungi and other fungal strains have also been documented [17,18]. Palmitic acid is also reported to display antioxidant, anti-atherosclerotic, and protein glycation inhibitory activities in a mouse model [19].

Phenolic and flavonoid compounds present in plants are known to be the major contributors to antioxidant activity [20]. The aqueous crude extract showed higher antioxidant activity compared to the methanol crude extract, which might be due to the higher concentrations of antioxidant compounds present. For example, catechol, which is a phenolic compound, was detected at a remarkably higher amount in the aqueous extract compared to the methanol extract. This compound is known to play an important role in scavenging free radicals and chelating transition metals [21]. Other antioxidant compounds were also detected in the methanol crude extract but only at lower concentrations - palmitic acid $(6.34 \%)$ and phytol $(1.75 \%)$, which were previously reported to have antioxidant activities [19,22]. The higher antioxidant activity of the aqueous extract compared to Trolox was highly encouraging, indicating that the extract has great potential as an antioxidant candidate. 
Apart from having antioxidant activity, phytol is also a phytochemical associated with chlorophyll metabolism and is shown to inhibit the growth of S. aureus and Mycobacterium tuberculosis [23-25]. In addition, it has shown antischistosomal, antinociceptive, antiinflammatory, and anti-allergic activities [22,26,27].

This study showed the phytochemical compositions of A. borneensis and its antimicrobial and antioxidant properties. Consequently, this ethnomedicinal plant, which has been traditionally used as an herbal tea in Brunei Darussalam, warrants further scientific investigation in order to fully evaluate its pharmacological status.

\section{CONCLUSION}

The phytochemical constituents of $A$. borneensis were found to have promising medicinal properties, and its extracts were found to have antimicrobial and antioxidant activities.

\section{ACKNOWLEDGMENTS}

The authors wish to acknowledge the support of the following grants: Brunei Research Council (JPKE/BRC/UBD/BRC6) and Universiti Brunei Darussalam (UBD/RSCH/1.13/ $\operatorname{FICBF}(b) / 2018 / 001)$.

\section{CONFLICT OF INTEREST}

The authors declare that they have no conflict of interests.

\section{REFERENCES}

1. Department of Agriculture. Medicinal plants of Brunei Darussalam. Department of Agriculture, Ministry of Industry and Primary Resources, Bandar Seri Begawan, Brunei Darussalam, 2000.

2. Universiti Brunei Darussalam. Medicinal plants of UBD botanical research centre. Universiti Brunei Darussalam, Brunei Darussalam, 2018.

3. Goh MPY, Basri AM, Yasin H, Taha H, Ahmad N. Ethnobotanical review and pharmacological properties of selected medicinal plants in Brunei Darussalam: Litsea elliptica, Dillenia suffruticosa, Dillenia excelsa, Aidia racemosa, Vitex pinnata and Senna alata. Asian Pac J Trop Biomed 2017;7(2):173-80.

4. Awang-Kamis ND, Taha H, Metali F. Effects of commercial plant hormones on the survival, rooting and growth of stem cuttings of an herbal tea plant, Aidia racemosa. Res J Med Plants 2016;10(6-7):414-9.

5. Metussin N, Mohamed H, Ahmad N, Yasin HM, Usman A. Evaluation of antioxidant capacity of Aidia borneensis leaf infusion, an endemic plant in Brunei Darussalam. Food Res 2018;2(1):12-9.

6. Diris MN, Basri AM, Metali F, Ahmad N, Taha H. Phytochemicals and antimicrobial activities of Melastoma malabathricum and Melastoma beccarianum leaf crude extracts. Res J Phytochemistry 2017;11(1):35-41.

7. Madaan R, Bansal G, Kumar S, Sharma A. Estimation of total phenols and flavonoids in extracts of Actaea spicata roots and antioxidant activity studies. Indian J Pharm Sci 2011;73(6):666-9.

8. Wu S, Xu T, Huang D. Chemical compositions of the volatile extracts from seeds of Dendranthema nankingense and Borago officinalis. J Food Drug Anal 2015;23(2):253-9.

9. RajashekarY, Raghavendra A, Bakthavatsalam N. Acetylcholinesterase inhibition by biofumigant (coumaran) from leaves of Lantana camara in stored grain and household insect pests. BioMed Res Int 2014; 2014:187019.

10. Inagaki M, Jyoyama $\mathrm{H}$, Ono $\mathrm{T}$, Yamada $\mathrm{K}$, Kobayashia M, Baba $\mathrm{T}$, et al. Synthesis and activities of oxidative metabolites of the anti- arthritic drug candidate S-2474. Bioorg Med Chem 2003;11(11): 2415-9.

11. Murakami M, Fukatsu K, Ohkawa S, Kasahara F, Sugawara T. ESR study on the antioxidant activity of TAK-218 in biological model membranes. Chem Pharm Bull 2000;48(6):784-92.

12. Kim MG, Lee HS. 1,2-benzendiol isolated from persimmon roots and its structural analogues show antimicrobial activities against food-borne bacteria. J Korean Soc Appl Biol Chem 2014;57(4): 429-33.

13. Jeong EY, Jeon JH, Lee CH, Lee HS. Antimicrobial activity of catechol isolated from Diospyros kaki Thunb. Roots and its derivatives toward intestinal bacteria. Food Chem 2009;115(3):1006-10.

14. Kocacaliskan I, Talan I, Terzi I. Antimicrobial activity of catechol and pyrogallol as allelochemicals. Z Naturforsch C Bio Sci 2006;61(910):639-42.

15. Basri AM, Taha H, Ahmad N. A review on the pharmacological activities and phytochemicals of Alpinia officinarum (Galangal) extracts derived from bioassay-guided fractionation and isolation. Pharmacogn Rev 2017;11(21):43-56.

16. Huang CB, Alimova Y, Myers TM, Ebersole JL. Short- and mediumchain fatty acids exhibit antimicrobial activity for oral microorganisms Arch Oral Biol 2011;56(7):650-4.

17. Altieri C, Cardillo D, Bevilacqua A, Sinigaglia M. Inhibition of Aspergillus spp. and Penicillium spp. by fatty acids and their monoglycerides. J Food Prot 2007;70(5):1206-12.

18. Liu S, Ruan W, Li J, Xu H. Biological control of phytopathogenic fungi by fatty acids. Mycopathologia 2008;166(2):93-102.

19. Cho KH, Hong JH, Lee KT. Monoacylglycerol (MAG)-oleic acid has stronger antioxidant, anti-atherosclerotic, and protein glycation inhibitory activities than MAG-palmitic acid. J Med Food 2010;13(1):99-107.

20. Nimse SB, Pal D. Free radicals, natural antioxidants and their reaction mechanisms. RSC Adv 2015;5:27986-8006.

21. Eshwarappa RSB, Iyer RS, Subbaramaiah SR, Richard SA, Dhananjaya BL. Antioxidant activity of Syzygium cumini leaf gall extracts. Bioimpacts 2014;4(2):101-7.

22. Santos CCMP, Salvadori MS, Mota VG, Costa LM, Almeida AACD, Oliveira GALD, et al. Antinociceptive and antioxidant activities of phytol in vivo and in vitro models. Neurosci J 2013;949452.

23. Inoue Y, Hada T, Shiraishi A, Hirose K, Hamashima H, Kobayashi S. Biphasic effects of geranylgeraniol, teprenone, and phytol on the growth of Staphylococcus aureus. Antimicrob Agents Chemother 2005;49(5):1770-4.

24. Rajab MS, Cantrell CL, Franzblau SG, Fischer NH. Antimycobacterial activity of (E)-phytol and derivatives: a preliminary structure-activity study. Planta Med 1998;64(1):2-4.

25. Saikia D, Parihar S, Chanda D, Ojha S, Kumar JK, Chanotiya CS, et al. Antitubercular potential of some semisynthetic analogues of phytol. Bioorg Med Chem Lett 2010;20(2):508-12.

26. Moraes JD, Oliveira RND, Costa JP, Junior ALG, Sousa DPD, Freitas RM, et al. Phytol, a diterpene alcohol from chlorophyll, as a drug against neglected tropical disease Schistosomiasis mansoni. PLoS Negl Trop Dis 2014;8(1):e2617.

27. Ryu KR, Choi JY, Chung S, Kim DH. Anti-scratching behavioral effect of the essential oil and phytol isolated from Artemisia princeps Pamp. in mice. Planta Med 2011;77(1):22-6.

How to cite this article:

Awang-Jamil Z, Basri AM, Ahmad N, Taha H. Phytochemical analysis, antimicrobial and antioxidant activities of Aidia borneensis leaf extracts. J Appl Biol Biotech 2019;7(05):92-97. 